\title{
Association between ALDH2 rs671 G>A polymorphism and gastric cancer susceptibility in Eastern Asia
}

\author{
You Jiang ${ }^{1}$, Jun Zhang ${ }^{1}$, Yuee $\mathrm{Wu}^{2}$, Jian $\mathrm{Wang}^{3}$ and Liang $\mathbf{L i}^{1}$ \\ ${ }^{1}$ Department of General Surgery, Hefei Second People's Hospital, Anhui Medical University, Hefei, Anhui, 230011, China \\ ${ }^{2}$ Department of Electrocardiogram Diagnosis, The Second Affiliated Hospital, Anhui Medical University, Hefei, Anhui, 230060, \\ China \\ ${ }^{3}$ Department of Pathology, The First Affiliated Hospital, Anhui Medical University, Hefei, Anhui, 230022, China
}

Correspondence to: Liang Li, email: 94355997@qq.com

Keywords: $A L D H 2$, polymorphism, meta-analysis, gastric cancer, risk

Received: August 18, $2017 \quad$ Accepted: September 20, 2017

Published: October 19, 2017

Copyright: Jiang et al. This is an open-access article distributed under the terms of the Creative Commons Attribution License 3.0 (CC BY 3.0), which permits unrestricted use, distribution, and reproduction in any medium, provided the original author and source are credited.

\section{ABSTRACT}

To date, the relationship between the aldehyde dehydrogenases-2 (ALDH2) rs671 G>A (Glu504Lys) polymorphism and gastric cancer (GC) risk has not been thoroughly elucidated. To derive a more precise estimation of the effect of the ALDH2 rs671 G>A polymorphism on GC, we conducted this meta-analysis. We searched for qualified studies in the Embase, PubMed, Wang Fan and China National Knowledge Infrastructure databases. Pooled odds ratios (ORs) and $95 \%$ confidence intervals (CIs) were calculated to assess the association. A total of 6,421 GC patients and 8,832 control subjects were included in the present study. The pooled results indicated no significant relationship between the ALDH2 rs671 G>A polymorphism and GC susceptibility in all genetic models. A stratified analysis by country showed that the ALDH2 rs671 G>A polymorphism might be a risk factor for GC in Japan (Allele model: $P_{\text {unadjusted }}=0.034$; Dominant model: $\left.P_{\text {unadjusted }}=0.040\right)$; however, the result was nonsignificant when the Bonferroni correction and false discovery rate (FDR) were applied. In subgroup analyses by drinking status in the dominant model, our study revealed that the ALDH2 rs671 G>A polymorphism significantly increased the risk of GC for drinkers (dominant model: $P<0.001$ ). No relationship between the ALDH2 rs671 G>A polymorphism and GC risk was observed in any other subgroup. Our present study indicated no association between the ALDH2 rs671 G>A polymorphism and GC risk in Eastern Asian populations. However, the ALDH2 rs671 G>A polymorphism can significantly increase GC risk for drinkers.

\section{INTRODUCTION}

Gastric cancer (GC) is a frequent malignant tumour and is one of the primary causes of tumour-associated deaths in the world. In 2012, 951,600 patients were diagnosed with $\mathrm{GC}$, accounting for $8 \%$ of cancer cases, and 723,100 patients died of GC, accounting for $10 \%$ of cancer deaths [1]. Recently, in many Western countries, the incidence of stomach cancer has gradually decreased. However, in Eastern Asia, the incidence and mortality rate of GC is rising, especially in China [2-4]. Gastric cancer has become a major public health problem, but the mechanism of carcinogenesis in gastric cancer is still unclear. It has been generally accepted that occurrence of stomach cancer is a multistep, complex and multifactorial process that involves diverse risk factors. Thus far, many environmental risk factors including drinking, smoking, Helicobacter pylori infection and micronutrient deficiency have been identified. Although people are exposed to the above factors, not all of them will develop GC, indicating that genetic factors are involved in the development of GC [5]. Single nucleotide polymorphisms (SNPs), the most frequent type of genetic mutations, may contribute to an individual's susceptibility to GC.

A SNP in the aldehyde dehydrogenase-2 (ALDH2) gene, rs671, is highly prevalent among the East Asian 
population and causes decreased ALDH2 enzyme activity which may result in an inability to eliminate acetaldehyde. Acetaldehyde, a metabolite of alcohol, is known to increase cancer susceptibility [6]. Polymorphisms of the human ALDH2 gene, which is located on chromosome 12q24, could alter blood acetaldehyde concentrations after alcohol intake [7]. The rs671 polymorphism (also called Glu487Lys) has been the most commonly investigated [8]. Several published studies that included East Asian populations have shown that the ALDH2 rs671 G>A polymorphism is associated with an increased risk of stomach cancer [9-11], and studies from Japan and Korea have indicated a possible interaction between alcohol intake and the ALDH2 rs671 polymorphism in the incidence of stomach cancerr $[9,10]$. ALDH2 polymorphism genotype frequencies are diverse among different ethnic populations [12]. The frequency of the ALDH2 rs671 A allele is very high in East Asians, and has not been observed in Africans, Caucasians, and Southeast Asians [13]. However, in East Asians, however, the frequency of the ALDH2 allele also varies among the Chinese, Koreans, and Japanese [14].

Previous case-control studies have reported a correlation between the ALDH2 rs671 G>A polymorphism and the development of stomach cancer, However, the results are still discrepant $[9,10,15]$. To clarify these findings, Cai et al. [16] conducted a metaanalysis of the associations between the ALDH2 rs671 $\mathrm{G}>\mathrm{A}$ polymorphism and cancer. Their study showed a significantly increased risk for GC associated with the ALDH2 rs671 G>A polymorphism, but there were only three case-control studies analysing the relationship between the ALDH2 rs671 G>A polymorphism and stomach cancer in the stratified analysis. One metaanalysis by Wang et al. [17] also indicated an increased risk for GC associated with the ALDH2 rs671 A allele; however, they included just two studies in the subgroup analysis of their meta-analysis on ALDH2 rs671 G>A. Another meta-analysis conducted by Mocellin et al. [18] suggested the opposite result; there was no relationship between the ALDH2 rs671 G>A polymorphism and the occurrence of stomach cancer. The sample sizes of these three meta-analyses are extremely small and their results are conflicting. Therefore, to more accurately assess the correlation, we decided to conduct a meta-analysis on all eligible case-control studies. Furthermore, we performed several subgroup analyses stratified by country, source of controls, sex, smoking status and drinking status.

\section{RESULTS}

\section{Characteristics of eligible studies}

Figure 1 shows a flow diagram of the study search process in our meta-analysis. The initial literature search identified 67 studies based on the selection strategy.
Fifty-six studies were left after the removal of duplicate studies. Thirty-five studies were excluded after reviewing the titles and abstracts. Among these 35 studies, 20 were not relevant studies, 5 were meta-analyses or reviews, 6 were not relevant to GC, 3 were not case-control studies, and 1 was not a human study. The full text of the rest of the studies was reviewed, and 9 of these full-text studies were removed for the following reasons: 4 studies did not have sufficient data, 1 had data covered by another study, 1 had data that overlapped with another, and 3 were not relevant to the ALDH2 rs671 G>A polymorphism. Finally, 12 case-control studies about the ALDH2 rs671 G>A polymorphism and GC risk were eventually included for further analysis, encompassing a total of 6,421 cases and 8,832 control subjects [9, 10, 15, 19-27]. Table 1 shows the characteristics of eligible studies. All the cases were histologically confirmed in each of the included studies. All 12 case-control studies were performed with Asians and were published from 2009 to 2017. Among these studies, 8 were conducted in China, 2 were in Japan and 2 were in Korea. Six studies were hospital-based and six were population-based. All control subjects in the selected studies were within Hardy-Weinberg equilibrium (HWE), except for those in Yuan's study [21]. The relationship between the ALDH2 rs671 G>A polymorphism and GC risk in subgroups according to drinking status, sex and smoking status was assessed in 7,2, and 2 studies, respectively. However, the author provided only the GG and GA + AA genotype counts of cases and controls without specific information on either the AA or GA genotype; thus, the dominant model was employed to assess the association between the ALDH2 rs671 G>A polymorphism and GC risk in the stratified analysis according to drinking status, sex and smoking status. The selected articles, assessed by the Newcastle-Ottawa Scale (NOS) score, ranged from 7 to 8 (Supplementary Table 1). The methodological quality of the included studies was reliable.

\section{Meta-analysis results}

Table 2 shows the allele frequency and genotype distribution of the ALDH2 $\mathrm{rs} 671 \mathrm{G}>$ A polymorphism in cases and controls. Table 3 shows the main results of our meta-analysis, which contained a total of 12 case-control studies with 6,421 GC patients and 8,832 control subjects. Our meta-analysis revealed no significant association between the ALDH2 rs671 $\mathrm{G}>\mathrm{A}$ polymorphism and $\mathrm{GC}$ risk in the overall pooled populations under any genetic model: AA VS GG (Odds ratio $(\mathrm{OR})=1.101$, $95 \%$ confidence interval $(\mathrm{CI})=0.924-1.313, I^{2}=23.2 \%$, $\left.P_{\text {unadjusted }}=0.282, P_{\text {Bonferroni }}=1.000, P_{\mathrm{FDR}}=0.461\right) ; \mathrm{GA}$ VS GG $\left(\mathrm{OR}=1.030,95 \% \mathrm{CI}=0.959-1.106, I^{2}=12.7 \%\right.$, $\left.P_{\text {unadjusted }}=0.423, P_{\text {Bonferroni }}=1.000, P_{\text {FDR }}=0.461\right)$; allele model $\left(\mathrm{OR}=1.031,95 \% \mathrm{CI}=0.972-1.093, I^{2}=10.9 \%\right.$, $\left.P_{\text {unadjusted }}=0.310, P_{\text {Bonferroni }}=1.000, P_{\mathrm{FDR}}=0.461\right)$; recessive model $(\mathrm{OR}=1.067,95 \% \mathrm{CI}=0.898-1.268$, 
$I^{2}=20.6 \%, P_{\text {unadjusted }}=0.461, P_{\text {Bonferroni }}=1.000, P_{\mathrm{FDR}}$ $=0.461)$; and dominant model $(\mathrm{OR}=1.033,95 \%$ $\mathrm{CI}=0.963-1.107, I^{2}=13.8 \%, P_{\text {unadjusted }}=0.365, P$ Bonferroni $\left.=1.000, P_{\text {FDR }}=0.461\right)$. The heterogeneity of the included studies was low in all genetic models, and the fixed effect model was applied. The stratified analysis subgroup by country revealed an increased GC risk in Japan based on the allele model (OR $=1.138,95 \%$ CI $=1.010-1.281, \mathrm{P}=0.034, I 2=0.0 \%$; Figure 2 ) and the dominant model $(\mathrm{OR}=1.172,95 \% \mathrm{CI}=1.008-1.364$, $\mathrm{P}=0.04, I 2=0.0 \%$; Figure 3 ), however, the result was not stable when the Bonferroni correction and false discovery rate (FDR) were applied (Allele model: $P$ Bonferroni $=0.170, P_{\mathrm{FDR}}=0.100$; Dominant model: $P_{\text {Bonferroni }}$ $=0.200, P_{\mathrm{FDR}}=0.100 ;$ Table 3 ). There was no statistically significant association between the ALDH2 rs671 G $>$ A polymorphism and GC risk in China and Korea in any genetic model (Table 3 ). No correlation between the
ALDH2 rs671 G > A polymorphism and susceptibility to GC was found in the hospital-based and population-based subgroups under any genetic model (Table 3). When sex, smoking status and drinking status subjects were analysed in the dominant model (GA+AA vs. GG), the ALDH2 rs671 $\mathrm{G}>\mathrm{A}$ polymorphism significantly increased the risk of GC for drinkers (dominant model: $\mathrm{OR}=1.421$, $95 \%$ $\mathrm{CI}=1.211-1.667, P<0.001, I^{2}=22.5 \%$; Figure 4$)$, and there was no statistically significant association between the ALDH2 rs671 $\mathrm{G}>\mathrm{A}$ polymorphism and GC in any other subgroup (Table 4 Supplementary Table 2).

\section{Sensitivity analyses}

All the included studies were in accordance with HWE in their controls except Yuan's, as shown in Table 1. To observe the impact of each single study on the pooled risk estimates, sensitivity analysis was performed with the

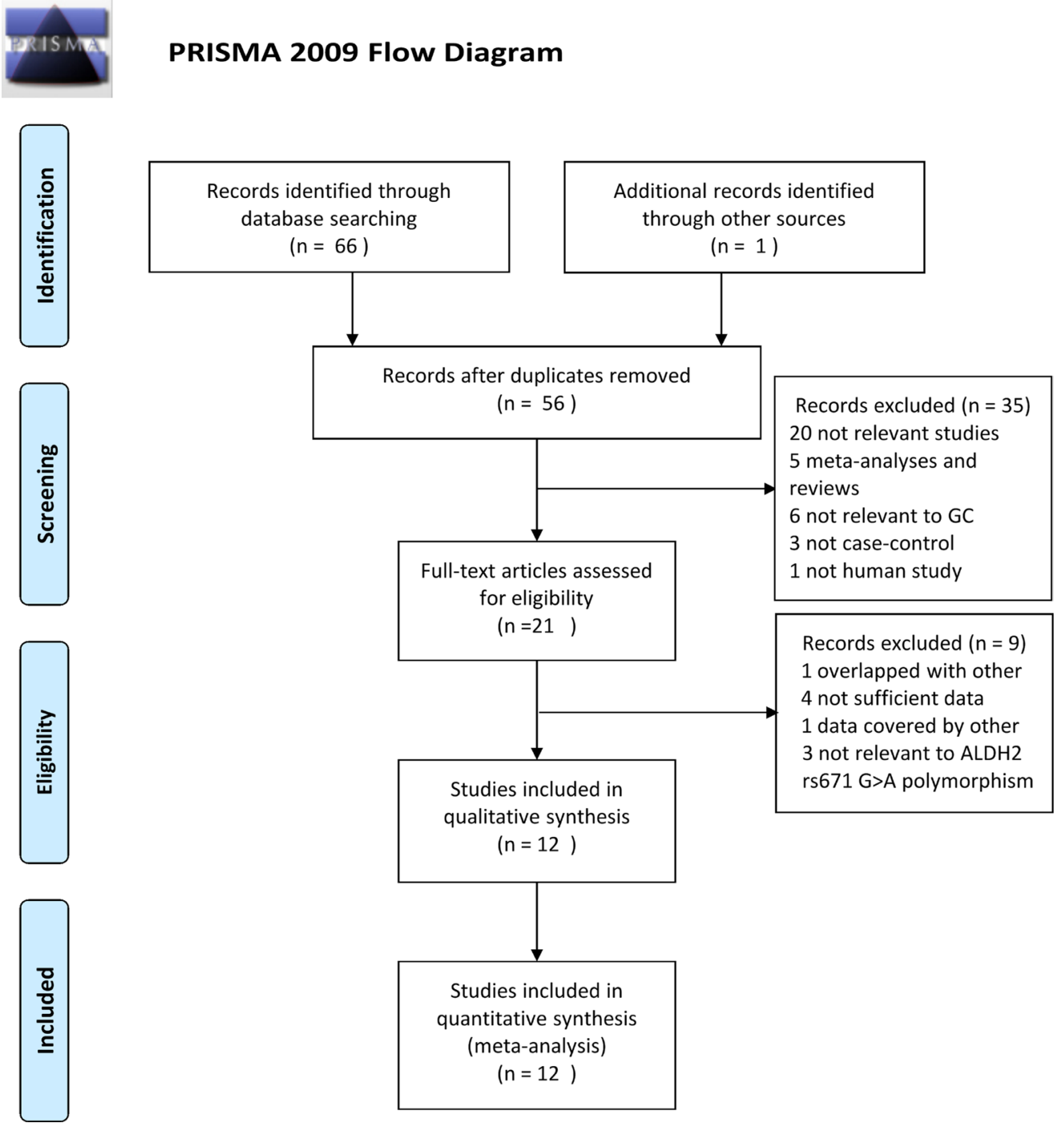

Figure 1: Flow chart of studies selection in this meta-analysis. 
Table 1: Characteristics of eligible case-control studies included in this meta-analysis

\begin{tabular}{lcccccccc}
\hline First author & Year & Country & Ethnicity & $\begin{array}{c}\text { Source of } \\
\text { controls }\end{array}$ & $\begin{array}{c}\text { Genotyping } \\
\text { method }\end{array}$ & $\begin{array}{c}\text { Number } \\
\text { (case/control) }\end{array}$ & HWE & $\begin{array}{c}\text { NOS } \\
\text { score }\end{array}$ \\
\hline Zhao et al. [19] & 2014 & China & Asians & HB & MALDI-TOFMS & $308 / 308$ & Yes & 7 \\
Li et al. [20] & 2009 & China & Asians & PB & PCR-DHPLC & $192 / 191$ & Yes & 8 \\
Zhou et al. [15] & 2010 & China & Asians & PB & PCR-DHPLC & $201 / 199$ & Yes & 8 \\
Yuan et al. [21] & 2016 & China & Asians & PB & PCR-DHPLC & $161 / 161$ & No & 8 \\
Hidaka et al. [22] & 2015 & Japan & Asians & PB & TaqMan & $457 / 457$ & Yes & 8 \\
Yang et al. [23] & 2016 & Korea & Asians & HB & MassARRAY & $450 / 1050$ & Yes & 7 \\
Cao et al. [24] & 2010 & China & Asians & PB & PCR-DHPLC & $382 / 382$ & Yes & 8 \\
Chen et al. [25] & 2016 & China & Asians & HB & PCR-RFLP & $246 / 274$ & Yes & 7 \\
Zhang et al. [26] & 2017 & China & Asians & HB & TaqMan & $2686 / 3675$ & Yes & 7 \\
Matsuo et al. [10] & 2013 & Japan & Asians & HB & TaqMan & $697 / 1372$ & Yes & 7 \\
Chang et al. [27] & 2014 & China & Asians & PB & PCR-RFLP & $196 / 393$ & Yes & 8 \\
Shin et al. [9] & 2011 & Korea & Asians & HB & PCR-RFLP & $445 / 370$ & Yes & 8 \\
\hline
\end{tabular}

PB: Population-based; HB: Hospital-based; HWE: Hardy-Weinberg equilibrium; NOS: Newcastle-Ottawa Scale; PCRRFLP: polymerase chain reaction-restriction fragment length polymorphism; MALDI-TOF-MS: polymerase chain reactionmatrix-assisted laser desorption/ionization time-of-flight mass spectrometry; DHPLC: denaturing high performance liquid chromatography.

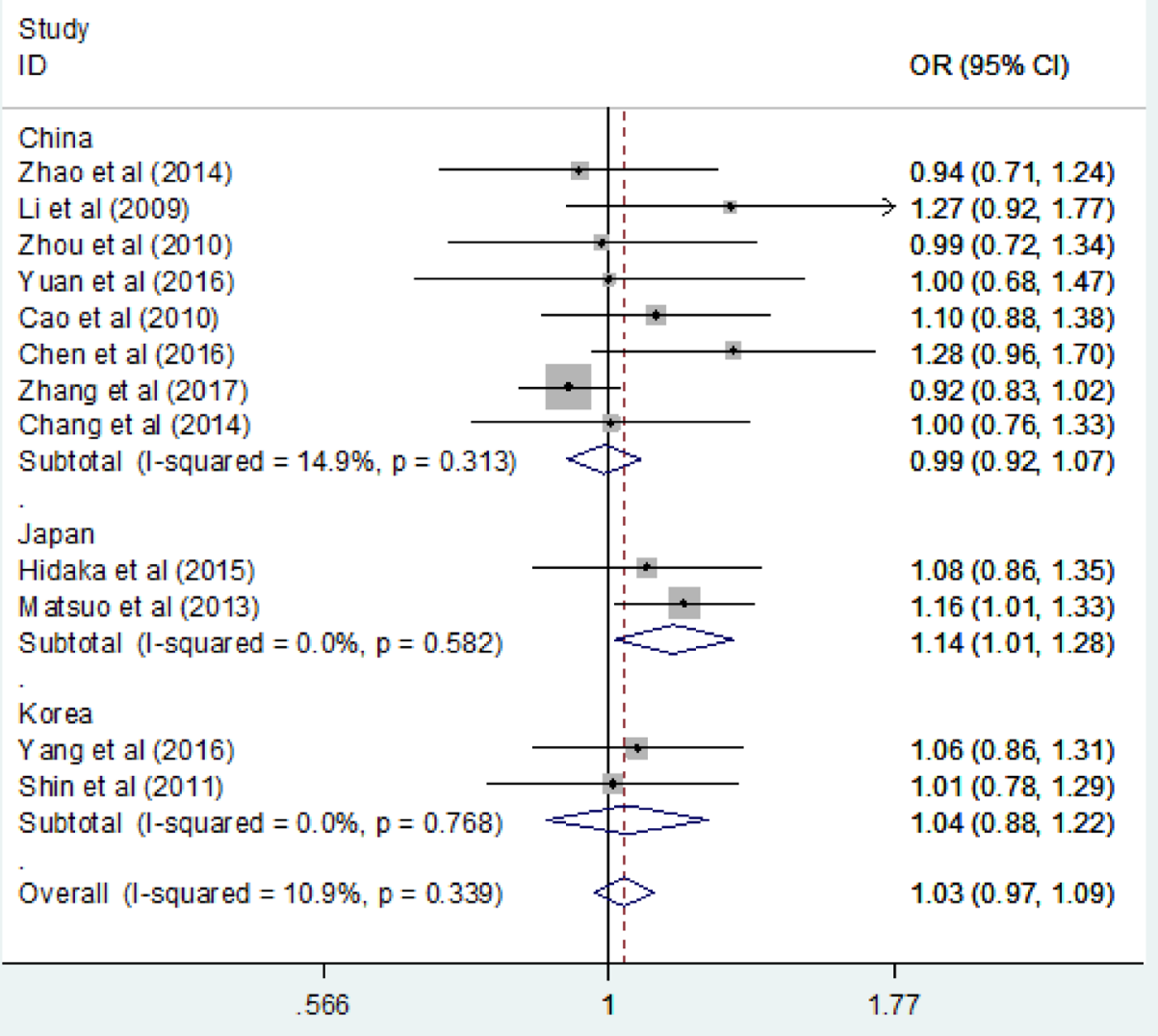

Figure 2: Forest plots of the ALDH2 rs671 G > A polymorphism and gastric cancer risk in subgroup by country (allele model: A vs. G). 
Table 2: ALDH2 (rs671) polymorphisms genotype distribution and allele frequency in cases and controls

\begin{tabular}{|c|c|c|c|c|c|c|c|c|c|c|c|c|c|c|c|}
\hline \multirow{3}{*}{ First author } & \multirow{3}{*}{ Year } & \multicolumn{8}{|c|}{ Genotype(N) } & & & & & \multirow{3}{*}{ MAF } & \multirow{3}{*}{ HWE } \\
\hline & & \multicolumn{4}{|c|}{ Case } & \multicolumn{4}{|c|}{ Control } & \multicolumn{2}{|c|}{ Case } & \multicolumn{2}{|c|}{ Control } & & \\
\hline & & Total & GG & GA & $\mathbf{A A}$ & Total & GG & GA & $\mathbf{A A}$ & G & $\mathbf{A}$ & G & $\mathbf{A}$ & & \\
\hline Zhao et al. [18] & 2014 & 308 & 194 & 106 & 8 & 308 & 194 & 100 & 14 & 494 & 122 & 488 & 128 & 0.21 & 0.81 \\
\hline Li et al. [19] & 2009 & 192 & 101 & 76 & 15 & 191 & 114 & 66 & 11 & 278 & 106 & 294 & 88 & 0.48 & 0.72 \\
\hline Zhou et al. [15] & 2010 & 201 & 99 & 91 & 11 & 199 & 97 & 91 & 11 & 289 & 113 & 285 & 113 & 0.28 & 0.08 \\
\hline Yuan et al. [20] & 2016 & 161 & 104 & 50 & 7 & 161 & 99 & 60 & 2 & 258 & 64 & 258 & 64 & 0.19 & 0.03 \\
\hline Hidaka et al. [21] & 2015 & 457 & 287 & 149 & 21 & 457 & 292 & 150 & 15 & 723 & 191 & 734 & 180 & 0.20 & 0.42 \\
\hline Yang et al. [22] & 2016 & 450 & 304 & 141 & 5 & 1050 & 736 & 292 & 22 & 749 & 151 & 1764 & 336 & 0.23 & 0.26 \\
\hline Cao et al. [23] & 2010 & 382 & 196 & 161 & 25 & 382 & 206 & 155 & 21 & 553 & 211 & 567 & 197 & 0.26 & 0.24 \\
\hline Chen et al. [24] & 2016 & 246 & 133 & 95 & 18 & 274 & 163 & 101 & 10 & 361 & 131 & 427 & 121 & 0.22 & 0.24 \\
\hline Zhang et al. [25] & 2017 & 2686 & 1995 & 643 & 48 & 3675 & 2663 & 941 & 71 & 4633 & 739 & 6267 & 1083 & 0.15 & 0.25 \\
\hline Matsuo et al. [10] & 2013 & 696 & 310 & 323 & 63 & 1372 & 683 & 580 & 109 & 943 & 449 & 1946 & 798 & 0.29 & 0.36 \\
\hline Chang et al. [26] & 2014 & 196 & 108 & 76 & 12 & 393 & 213 & 160 & 20 & 292 & 100 & 586 & 200 & 0.25 & 0.15 \\
\hline Shin et al. [9] & 2011 & 445 & 291 & 141 & 13 & 370 & 250 & 102 & 18 & 723 & 167 & 602 & 138 & 0.19 & 0.08 \\
\hline
\end{tabular}

MAF: minor allele frequency; HWE: Hardy-Weinberg equilibrium.

Study

ID

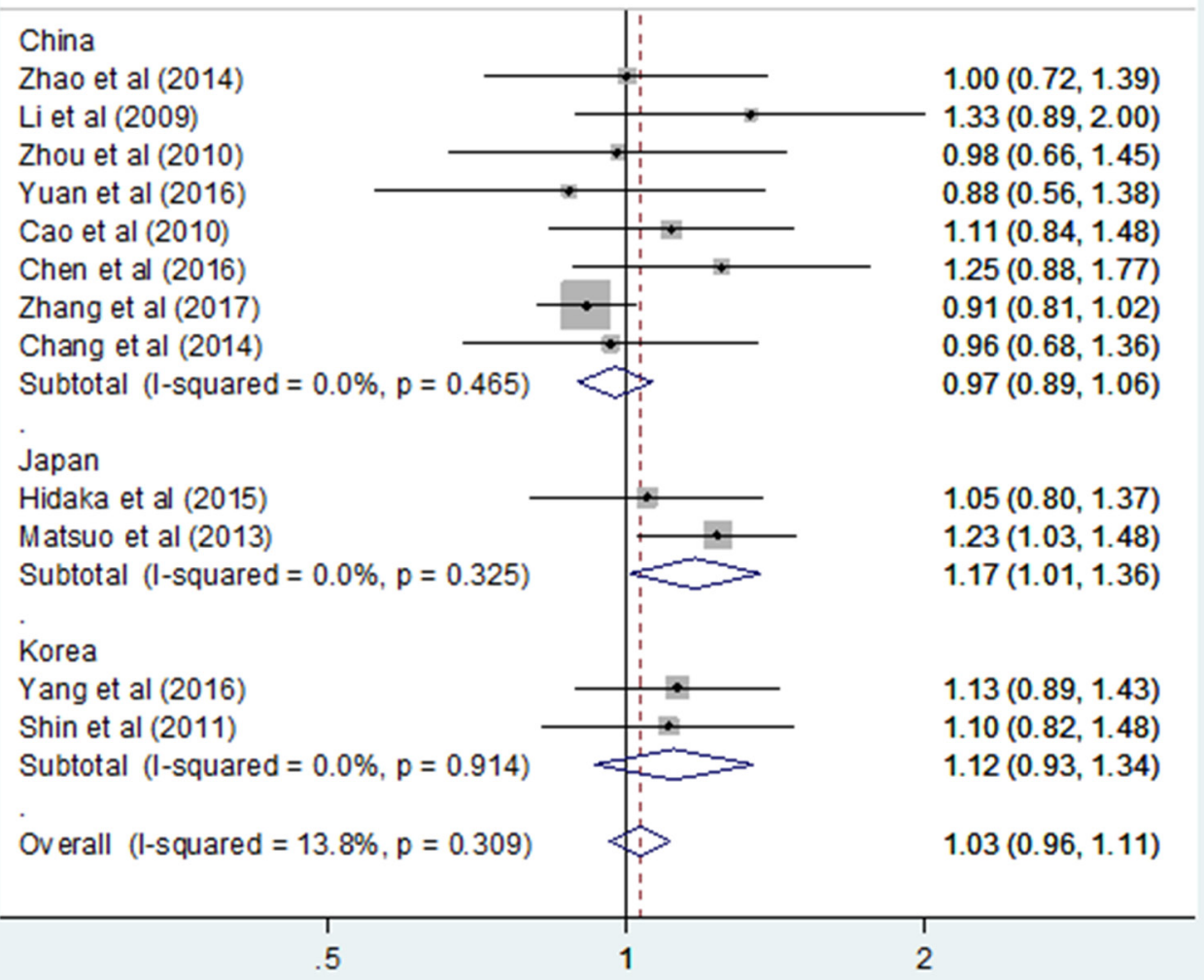

Figure 3: Forest plots of the ALDH2 rs671 G > A polymorphism and gastric cancer risk in subgroup by country (dominant model: GA + AA vs. GG). 
leave-one-out cross-validation method. When removing any individual article from the analysis, the overall outcomes did not significantly change in the pooled ORs of the overall GC risk, indicating that our meta-analysis is credible and reliable (Figure 5, data not shown).

\section{Publication bias}

Begg's funnel plot and Egger's test were used to evaluate publishing bias. Figure 6 shows that the funnel plot demonstrated no apparent asymmetry, suggesting there is no significant publication bias in the overall population. In our present meta-analysis, there was no evidence of publication bias observed by Begg's test and Egger's test (Table 3, Figure 6).

\section{DISCUSSION}

ALDH2 is the main enzyme responsible for the metabolism of acetaldehyde, which increases cancer risk [28]. Several mutations have been confirmed in the human ALDH2 gene, including rs671 G>A, rs 16941667 $\mathrm{C}>\mathrm{T}$, rs968529 $\mathrm{C}>\mathrm{T}$ and $\mathrm{rs} 886205 \mathrm{~T}>\mathrm{C}[16,29]$. Among these alleles, the ALDH2 gene rs671 G>A, namely, the Glu504Lys polymorphism, is the most widely investigated and potentially functional. Several genomewide association (GWA) studies have demonstrated that the ALDH2 gene rs671 G>A polymorphism can significantly increase the risk of some tumours, including upper aerodigestive tract (UADT) cancer and oesophageal squamous cell carcinoma (OSCC) [30, 31]. In recent years, an accumulation of published studies have investigated the relationship between the ALDH2 rs671 $\mathrm{G}>\mathrm{A}$ polymorphism and the risk of $\mathrm{GC}$; however, the results are still conflicting $[9,10]$. Therefore, to make a more accurate assessment of the correlation, we decided to perform the present meta-analysis on all eligible casecontrol studies.

Our meta-analysis included a total of 12 case-control studies with 6,421 GC patients and 8,832 control subjects and showed that there was no significant association between the ALDH2 rs671 G>A polymorphism and GC risk in the overall pooled population under all five models. GWAS plays an important role in the procedures for the discovery of genetic mutations and the reliability of metaanalysis. However, there are no previous relevant GWAS

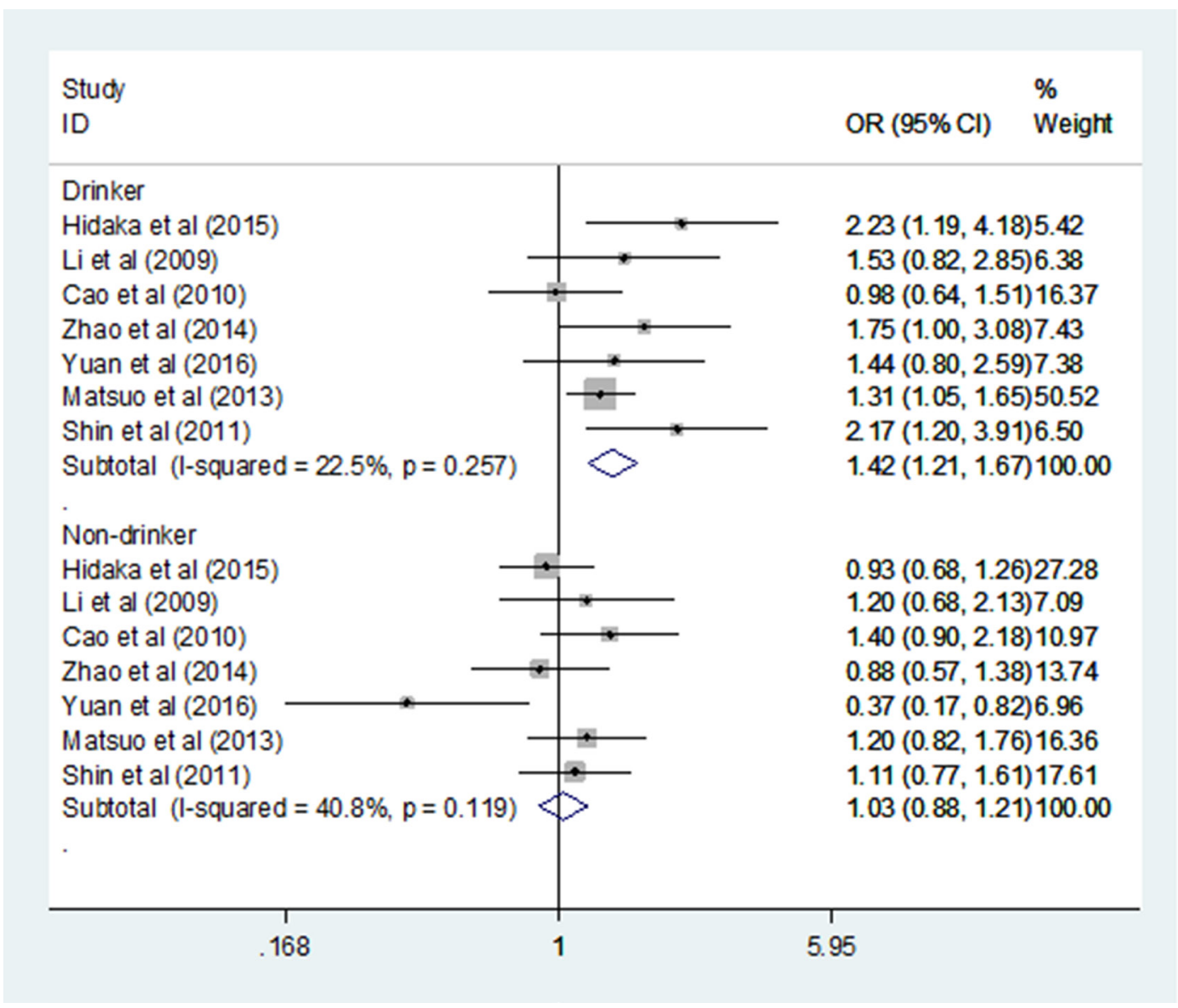

Figure 4: Forest plots of the ALDH2 rs671 G>A polymorphism and gastric cancer risk in the Drinker and Nonsmoker subgroup (dominant model: GA+AA vs. GG). 
Table 3: Meta-analysis results

\begin{tabular}{|c|c|c|c|c|c|c|c|c|c|}
\hline \multirow{2}{*}{$\begin{array}{l}\text { Genetic } \\
\text { model }\end{array}$} & \multirow{2}{*}{ Category } & \multirow{2}{*}{ OR $(95 \%$ CI $)$} & \multirow{2}{*}{$P$} & \multirow{2}{*}{ Bon } & \multirow{2}{*}{ FDR } & \multicolumn{2}{|c|}{ Heterogeneity } & \multirow{2}{*}{$\begin{array}{c}\begin{array}{c}\text { Begg's } \\
\text { test }\end{array} \\
P\end{array}$} & \multirow{2}{*}{$\begin{array}{c}\begin{array}{c}\text { Egger } \\
\text { test }\end{array} \\
P\end{array}$} \\
\hline & & & & & & $I^{2}$ & $\boldsymbol{P}$ & & \\
\hline \multirow[t]{6}{*}{ AA VS GG } & Overall & $1.101[0.924 ; 1.313]$ & 0.282 & 1.000 & 0.461 & $23.2 \%$ & 0.215 & 0.837 & 0.872 \\
\hline & China & $1.110[0.879 ; 1.403]$ & 0.380 & 1.000 & 0.693 & $20.4 \%$ & 0.267 & & \\
\hline & Japan & $1.303[0.963 ; 1.763]$ & 0.087 & 0.435 & 0.109 & $0.0 \%$ & 0.773 & & \\
\hline & Korea & $0.592[0.329 ; 1.064]$ & 0.080 & 0.400 & 0.155 & $0.0 \%$ & 0.847 & & \\
\hline & HB & $0.592[0.329 ; 1.064]$ & 0.926 & 1.000 & 0.926 & $52.4 \%$ & 0.062 & & \\
\hline & PB & $0.592[0.329 ; 1.064]$ & 0.074 & 0.370 & 0.220 & $0.0 \%$ & 0.845 & & \\
\hline \multirow[t]{6}{*}{ GA VS GG } & Overall & $1.030[0.959 ; 1.106]$ & 0.423 & 1.000 & 0.461 & $12.7 \%$ & 0.321 & 0.537 & 0.240 \\
\hline & China & $0.965[0.883 ; 1.055]$ & 0.431 & 1.000 & 0.693 & $0.0 \%$ & 0.611 & & \\
\hline & Japan & $1.152[0.985 ; 1.350]$ & 0.076 & 0.380 & 0.109 & $20.8 \%$ & 0.261 & & \\
\hline & Korea & $1.176[0.973 ; 1.422]$ & 0.093 & 0.465 & 0.155 & $0.0 \%$ & 0.937 & & \\
\hline & HB & $1.033[0.952 ; 1.122]$ & 0.435 & 1.000 & 0.926 & $48.6 \%$ & 0.083 & & \\
\hline & $\mathrm{PB}$ & $1.019[0.882 ; 1.176]$ & 0.801 & 1.000 & 0.801 & $0.0 \%$ & 0.726 & & \\
\hline \multirow[t]{6}{*}{ Allele model } & Overall & $1.031[0.972 ; 1.093]$ & 0.310 & 1.000 & 0.461 & $10.9 \%$ & 0.339 & 0.945 & 0.185 \\
\hline & China & $0.991[0.921 ; 1.067]$ & 0.815 & 1.000 & 0.815 & $14.9 \%$ & 0.313 & & \\
\hline & Japan & $1.138[1.010 ; 1.281]$ & 0.034 & 0.170 & 0.100 & $0.0 \%$ & 0.582 & & \\
\hline & Korea & $1.037[0.883 ; 1.218]$ & 0.659 & 1.000 & 0.659 & $0.0 \%$ & 0.768 & & \\
\hline & HB & $1.016[0.949 ; 1.088]$ & 0.647 & 1.000 & 0.926 & $49.9 \%$ & 0.076 & & \\
\hline & PB & $1.072[0.957 ; 1.200]$ & 0.229 & 1.000 & 0.381 & $0.0 \%$ & 0.884 & & \\
\hline \multirow[t]{6}{*}{$\begin{array}{l}\text { Recessive } \\
\text { model }\end{array}$} & Overall & $1.067[0.898 ; 1.268]$ & 0.461 & 1.000 & 0.461 & $20.6 \%$ & 0.241 & 0.745 & 0.813 \\
\hline & China & $1.107[0.878 ; 1.395]$ & 0.389 & 1.000 & 0.693 & $15.6 \%$ & 0.308 & & \\
\hline & Japan & $1.200[0.897 ; 1.607]$ & 0.220 & 1.000 & 0.220 & $0.0 \%$ & 0.587 & & \\
\hline & Korea & $0.563[0.314 ; 1.008]$ & 0.053 & 0.265 & 0.155 & $0.0 \%$ & 0.854 & & \\
\hline & HB & $0.973[0.790 ; 1.198]$ & 0.799 & 1.000 & 0.926 & $47.6 \%$ & 0.089 & & \\
\hline & $\mathrm{PB}$ & $1.312[0.960 ; 1.793]$ & 0.088 & 0.440 & 0.220 & $0.0 \%$ & 0.825 & & \\
\hline \multirow[t]{6}{*}{$\begin{array}{c}\text { Dominant } \\
\text { model }\end{array}$} & Overall & $1.033[0.963 ; 1.107]$ & 0.365 & 1.000 & 0.461 & $13.8 \%$ & 0.309 & 0.537 & 0.163 \\
\hline & China & $0.974[0.894 ; 1.062]$ & 0.554 & 1.000 & 0.693 & $0.0 \%$ & 0.465 & & \\
\hline & Japan & $1.172[1.008 ; 1.364]$ & 0.040 & 0.200 & 0.100 & $0.0 \%$ & 0.325 & & \\
\hline & Korea & $1.116[0.928 ; 1.342]$ & 0.365 & 1.000 & 0.456 & $0.0 \%$ & 0.914 & & \\
\hline & HB & $1.026[0.947 ; 1.112]$ & 0.526 & 1.000 & 0.926 & $51.0 \%$ & 0.070 & & \\
\hline & PB & $1.052[0.916 ; 1.208]$ & 0.476 & 1.000 & 0.595 & $0.0 \%$ & 0.781 & & \\
\hline
\end{tabular}

OR: odds ratio; 95\% CI: 95\% confidence interval; Bon: $P$-value in Bonferroni testing; FDR: false discovery rate.

investigating the relationship between the ALDH2 rs671 $\mathrm{G}>\mathrm{A}$ polymorphism and the GC risk. Three previous metaanalyses assessed the association of the ALDH2 rs671 G>A polymorphism and GC susceptibility $[16,17,18]$. One meta-analysis performed in 2014 by Wang et al. [17] included two case-control studies with a total of 1,079 cases and 1,754 controls. Their study indicated that the ALDH2 rs671 G>A polymorphism significantly increased the risk of GC. Another meta-analysis carried out in 2015 by Cai et al. [16] included three case-control studies with a total of 1,523 cases and 2,124 controls in the subgroup analysis. Their results also indicated a statistically significant association between the ALDH2 rs671 G>A polymorphism and GC risk. Both results were inconsistent with the findings of our meta-analysis. These conflicting findings are likely the result of the sample sizes and diverse genetic backgrounds. The third meta-analysis conducted in 2015 by Mocellin et al. [18] showed no relationship between ALDH2 rs671 G>A polymorphism and GC risk. Although their results were consistent with our study, the sample size in their study was 


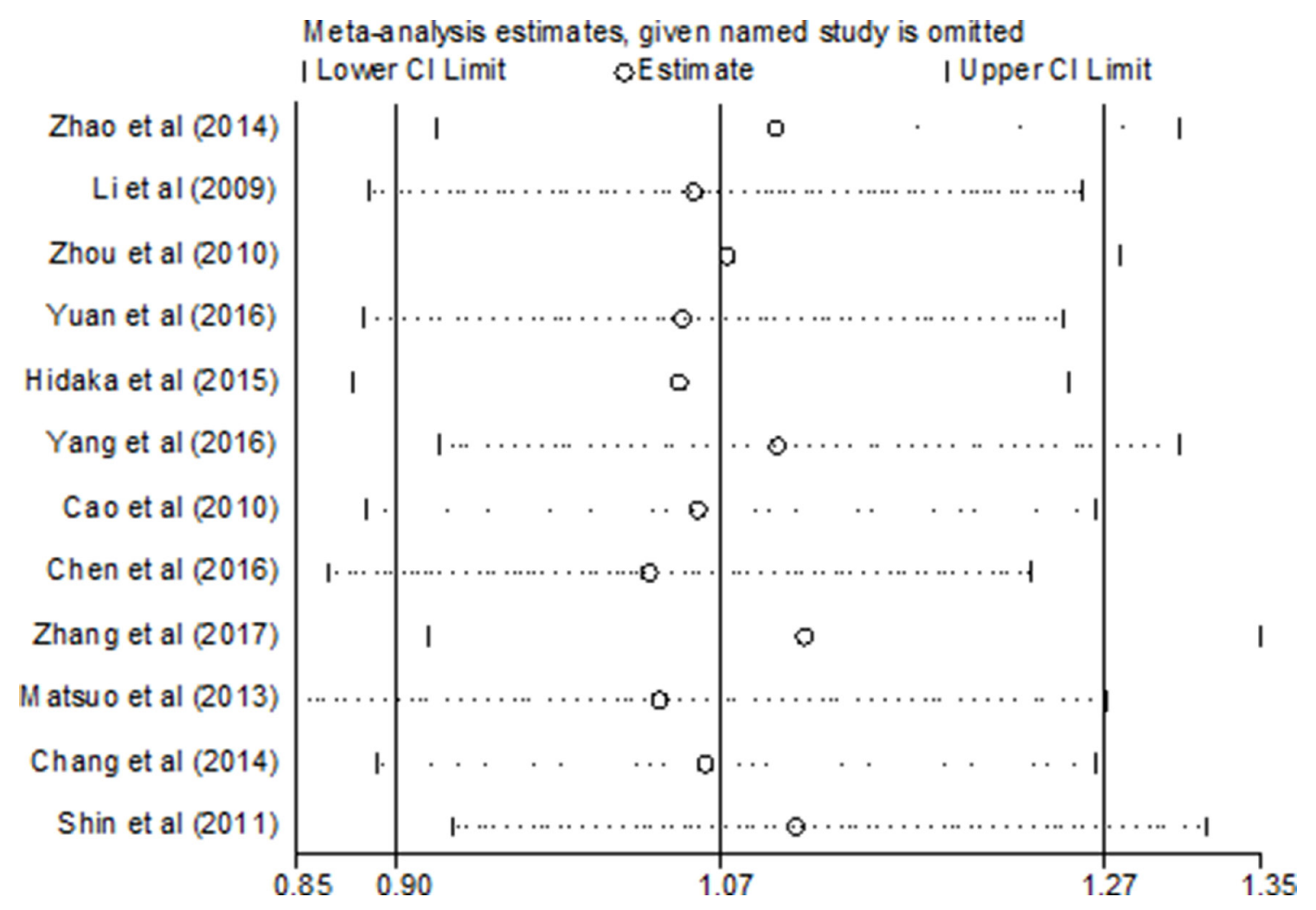

Figure 5: Sensitivity analysis of the ALDH2 rs671 G > A polymorphism and gastric cancer risk (recessive model: AA vs. GA + GG).

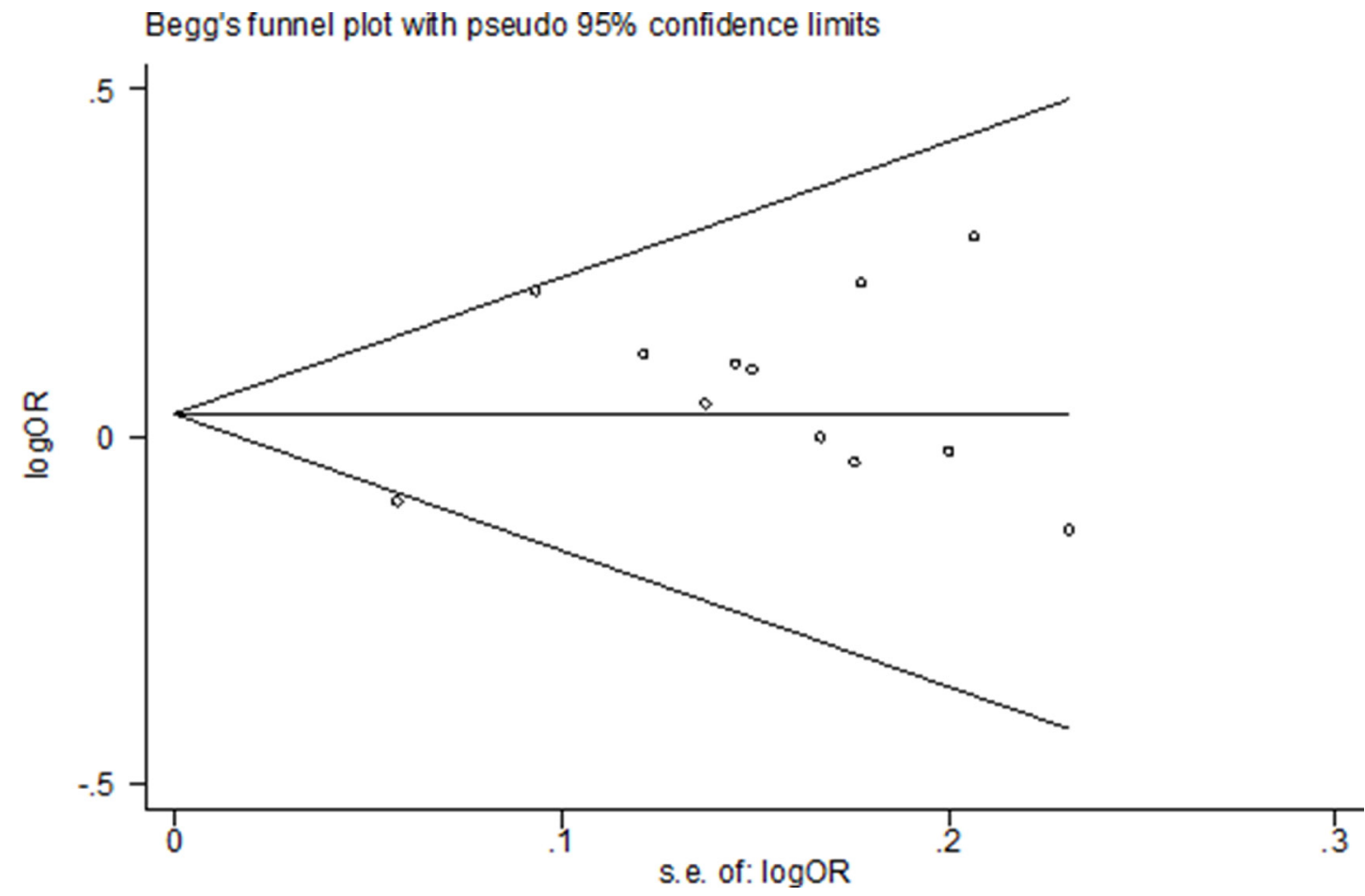

Figure 6: Funnel plot assessing evidence of publication bias from 12 studies (dominant model: GA+AA vs. GG). 
Table 4: Association between ALDH2 rs671 G>A polymorphism and sex, smoking status and drinking status of the gastric cancer patients based on dominant models

\begin{tabular}{lccccccc}
\hline & \multicolumn{7}{c}{ Dominant model: GA + AA vs. GG } \\
\cline { 2 - 7 } Subgroup analyses & OR & $\mathbf{9 5 \%}$ CI & P value & $\boldsymbol{I}$ & $\boldsymbol{P}_{\text {Het }}$ & $\begin{array}{c}\text { Effects } \\
\text { model }\end{array}$ & $\begin{array}{c}\text { No. of } \\
\text { studies }\end{array}$ \\
\cline { 2 - 8 } & & & & & & & \\
\hline Sex & 1.049 & $0.942-1.168$ & 0.385 & $93.9 \%$ & $<0.001$ & $\mathrm{R}$ & 2 \\
Male & 1.053 & $0.905-1.224$ & 0.506 & $0.0 \%$ & 0.980 & $\mathrm{~F}$ & 2 \\
Female & & & & & & & \\
Smoking status & 1.212 & $0.851-1.728$ & 0.286 & $0.0 \%$ & 0.612 & $\mathrm{~F}$ & 2 \\
Smoker & 0.599 & $0.400-0.897$ & 0.080 & $43.2 \%$ & 0.184 & $\mathrm{~F}$ & 2 \\
Nonsmoker & & & & & & & \\
Drinking status & 1.421 & $1.211-1.667$ & $<\mathbf{0 . 0 0 1}$ & $22.5 \%$ & 0.257 & $\mathrm{~F}$ & 7 \\
Drinker & 1.031 & $0.879-1.209$ & 0.707 & $40.8 \%$ & 0.119 & $\mathrm{~F}$ & 7 \\
Nondrinker & & & & & & & \\
\hline
\end{tabular}

F: fixed effects model; R: random effects model.

also extremely small, and the statistical power of their study was low. The results of our meta-analysis, which included more case-control studies, have more sufficient statistical power and are more reliable.

When stratified by country, the subgroup study indicated that the ALDH2 rs671 G>A polymorphism significantly increased the risk of GC in Japan based on the allele model and dominant model (Allele model: $P_{\text {unadjusted }}$ $=0.034$; Dominant model: $P_{\text {unadjusted }}=0.040$ ). There was no statistically significant relationship in China and Korea under any genetic models. This result suggests that the differences between countries might be a potential source of heterogeneity for this relationship. It is hypothesized that differences between countries might reflect diversity in alleles and genotypes among diverse ethnic populations. However, this result should be interpreted prudently and confirmed by more case-control studies, as the sample size in the Japan subgroup is extremely small. When the Bonferroni correction and FDR were applied to adjust for multiple comparisons, the result was unreliable and showed no association between the ALDH2 rs671 G>A polymorphism and GC risk in Japan under any models. No statistically significant relationships were found in the population-based and hospital-based subgroup according to the source of controls. When the subgroups were split up by sex, smoking status and drinking status in the dominant model, our study revealed that the ALDH2 rs671 $\mathrm{G}>\mathrm{A}$ polymorphism significantly increased the risk of $\mathrm{GC}$ for drinkers. The impact of the ALDH2 rs $671 \mathrm{G}>\mathrm{A}$ polymorphism on alcohol induced carcinogenesis has been identified in published studies of cancers of the UADT, including head and neck cancers and OSCC $[32,33]$. Several studies have reported the relationship between the ALDH2 rs671 G>A polymorphism and GC risk among alcoholics; however, the results are inconsistent. Cao et al.
[24] showed that the ALDH2 rs671 G>A polymorphism and alcohol drinking may not play an important role in the occurrence of gastric cancer. However, more studies have demonstrated the opposite result and obtained a positive result $[9,10,19,22]$. Our meta-analysis assessed the association of the ALDH2 rs671 G>A polymorphism, alcohol drinking and GC risk with seven case-control studies and showed that the risk of stomach cancer among drinkers was increased by 1.4-fold compared with that among non-drinkers. No significant association between the ALDH2 rs671 G>A polymorphism and stomach cancer risk was found in any other subgroups.

Although our study is the most up-to-date metaanalysis and included all eligible case-control studies until July 2017, similar to other studies, our study shares several flaws with other studies in the following aspects. First, although no obvious publication bias was shown in our study, some bias is unavoidable because only published studies were included. Some published studies in accordance with the conditions may not be included. Second, as a systematic summary of the data, our study did not demonstrate an association at the level of basic experiments. However, due to the finite number of selected studies and samples, the published data included in our study did not have a sufficiently large sample size for comprehensive analysis. Third, the selected papers in our study were mostly from the Chinese population. The number of case-control studies in certain stratified analyses was too small to acquire a reliable association. Finally, only the dominant model was applied to assess the relationship when stratifying by sex, smoking status and drinking status due to insufficient data. More original data from a large sample of multiple centres is needed to confirm the relationship between the ALDH2 rs671 G>A polymorphism and GC susceptibility. 
In conclusion, our meta-analysis showed no association between the ALDH2 rs671 G>A polymorphism and $\mathrm{GC}$ risk in Eastern Asian populations. The ALDH2 rs671 $\mathrm{G}>\mathrm{A}$ polymorphism and alcohol drinking had a synergistic interaction for GC risk. Data from a large sample of further investigations are still needed to confirm the roles of ALDH2 in GC and validate these associations.

\section{MATERIALS AND METHODS}

\section{Search strategy}

To screen out eligible studies, we searched relevant studies in the Embase, PubMed, Wang Fan and China National Knowledge Infrastructure (CNKI) databases. No language limitation was used in the search and the last search was updated on July 16, 2017. The following search key terms were used: ('ALDH2' or 'aldehyde' or 'dehydrogenases-2') and ('gastric carcinoma' or 'gastric cancer' or 'stomach cancer') and ('polymorphisms' or 'genotype' or 'polymorphism'). We also studied the reference lists of the included studies and recent reviews.

\section{Inclusion and exclusion criteria}

The eligible studies included in this meta-analysis must have provided the following information: (1) the relationship between the ALDH2 rs671 G>A polymorphism and GC risk; (2) a case-control study; (3) sufficient data for estimating an OR and the corresponding 95\% CI; and (4) GC diagnoses and the sources of cases and controls were clearly described in the study. Studies were excluded for the following reasons: (1) duplicate data; (2) insufficient data; and (3) abstracts, reviews, comments and editorial letters. The largest published studies were selected if the same or overlapping data were used.

\section{Extracted information}

According to the above inclusion criteria, two investigators independently extracted information from all collected studies. Discrepancies were solved by discussion among all investigators. We collected the following characteristics from each eligible study: the first author, year of publication, ethnicity, country, study design, source of cases, genotyping methods, number of cases and controls, minor allele frequency (MAF) in controls, matching variables, genotypes, source of the control group (population or hospital-based controls), evidence of HWE in the control group, and others.

\section{Quality assessment}

Two investigators independently assessed the quality of the included studies using the NOS. The NOS was performed to assess the study quality based on the following aspects: selection, comparability, and exposure situation in case-control studies. Rating scores ranged from 0 to 9 . Studies with a score higher than seven were considered to be of good quality (Supplementary Table 1).

\section{Statistical analysis}

HWE in the controls was assessed for each study with a goodness-of-fit test (Chi square or Fisher's exact test). HWE was considered to be in significant disequilibrium when $P$ was less than 0.05 . We assessed the strength of the association between the ALDH2 rs671 $\mathrm{G}>$ A polymorphism and GC susceptibility using ORs with 95\% CIs in the co-dominant model (AA vs. GG and GA vs. GG), recessive model (AA vs. GA+GG), dominant model (GA+AA vs. GG), and allele model (A vs. G). We conducted subgroup tests according to country, source of controls, sex, smoking status and drinking status. The significance of pooled ORs was tested using $\mathrm{Z}$ test. Differences were considered statistically significant when $P$ was less than 0.05 . The $I^{2}$ test was used to explore the heterogeneity among eligible studies [34]. The random (DerSimonian-Laird method) effect model was used to calculate the pooled OR when the $I^{2}$ value $>50 \%$ and was considered to represent significant statistical heterogeneity [35]. The fixed (Mantel-Haenszel method) effect model was used to measure the pooled OR when the $I^{2}$ value < $50 \%$ and was considered to represent less heterogeneity [36]. To explore the influence of each included study, a sensitivity analysis was performed by excluding one study at a time and then examining the pooled OR by repeating the meta-analysis. Publication bias was assessed using Begg's rank correlation method and Egger's weighted regression method $(P<0.05$ was considered statistically significant). Funnel plots were also used to illustrate the publication bias [37, 38]. All statistical analyses were performed using STATA 12.0 soft-ware (version 12.0; STATA Corp. College Station, TX, USA) using twotailed $P$-values. To adjust for multiple comparisons, the Bonferroni correction method and FDR were applied. The power of the meta-analysis for each polymorphism to detect some effect size was estimated according to the method recommended by Hedges and Pigott with $P<0.05$ considered as statistically significant [39].

\section{Author contributions}

You Jiang conceived and designed the study; Jun Zhang and Jian Wang collected the data; You Jiang and Yuee Wu analysed the data; and You Jiang and Liang Li contributed to the writing of the manuscript.

\section{ACKNOWLEDGMENTS}

We are indebted to all the people who helped with our study. 


\section{CONFLICTS OF INTEREST}

The authors declare that there were no conflicts of interest.

\section{REFERENCES}

1. Torre LA, Bray F, Siegel RL, Ferlay J, Lortet-Tieulent J, Jemal A. Global cancer statistics, 2012. CA Cancer J Clin. 2015; 65:87-108.

2. Karimi P, Islami F, Anandasabapathy S, Freedman ND, Kamangar F. Gastric cancer: Descriptive epidemiology, risk factors, screening and prevention. Cancer Epidemiol Biomarkers Prev. 2014; 23:700-713.

3. Jemal A, Bray F, Center MM, Ferlay J, Ward E, Forman D. Global cancer statistics. CA Cancer J Clin. 2011; 61: 69-90.

4. Yang L. Incidence and mortality of gastric cancer in China. World J Gastroenterol. 2006; 12: 17-20.

5. Woo HD, Lee J, Choi IJ, Kim CG, Lee JY, Kwon O, Kim J. Dietary flavonoids and gastric cancer risk in a Korean population. Nutrients. 2014; 6:4961-4973.

6. Yoshida A. Genetics of human alcohol-metabolizing enzymes. Progress in Nucleic Acid Research and Molecular Biology. 1991; 40:225-267.

7. Eriksson CJ. The role of acetaldehyde in the actions of alcohol (update 2000). Alcohol Clin Exp Res. 2001; $25: 15 \mathrm{~S}-32 \mathrm{~S}$.

8. Eng MY, Luczak SE, Wall TL. ALDH2, ADH1B, and ADH1C genotypes in Asians: a literature review. Alcohol Res Health. 2007; 30:22-27.

9. Shin CM, Kim N, Cho SI, Kim JS, Jung HC, Song IS. Association between alcohol intake and risk for gastric cancer with regard to ALDH2 genotype in the Korean population. International Journal of Epidemiology. 2011; 40:1047-1055.

10. Matsuo K, Oze I, Hosono S, Ito H, Watanabe M, Ishioka K, Ito S, Tajika M, Yatabe Y, Niwa Y. The aldehyde dehydrogenase 2 (ALDH2) Glu504Lys polymorphism interacts with alcohol drinking in the risk of stomach cancer. Carcinogenesis. 2013; 34:1510-1515.

11. Yokoyama A, Muramatsu T, Omori T, Yokoyama T, Matsushita S, Higuchi S, Maruyama K, Ishii H. Alcohol and aldehyde dehydrogenase gene polymorphisms and oropharyngolaryngeal, esophageal and stomach cancers in Japanese alcoholics. Carcinogenesis. 2001; 22:433-439.

12. Li DW, Zhao HY, Gelernter J. Strong protective effect of the aldehyde dehydrogenase gene (ALDH2) 504lys $(* 2)$ allele against alcoholism and alcohol-induced medical diseases in Asians. Hum Genet. 2012; 131:725-737.

13. Brennan P, Lewis S, Hashibe M, Bell DA, Boffetta P, Bouchardy C, Caporaso N, Chen C, Coutelle C, Diehl SR, Hayes RB, Olshan AF, Schwartz SM, et al. Pooled analysis of alcohol dehydrogenase genotypes and head and neck cancer: A huge review. Am J Epidemiol. 2004; 159:1-16.
14. Hamajima N, Takezaki T, Tajima K. Allele frequencies of 25 polymorphisms pertaining to cancer risk for Japanese, Koreans and Chinese. Asian Pac J Cancer Prev. 2002; 3:197-206.

15. Zhou ZY, Li JQ, Shun GX, Xu MG, Xu XY. Genetic polymorphisms of ADH2, ALDH2 and alcohol drinking investigated for their connection with stomach cancer. Shanghai J Prev Med. 2010; 22: 207-209.

16. Cai Q, Wu J, Cai Q, Chen EZ, Jiang ZY. Association between Glu504Lys Polymorphism of ALDH2 Gene and Cancer Risk: A Meta-Analysis. PLoS One. 2015; 10:e0117173.

17. Wang HL, Zhou PY, Liu P, Zhang Y. ALDH2 and ADH1 Genetic Polymorphisms May Contribute to the Risk of Gastric Cancer: A Meta-Analysis. PloS One. 2014; 9:e88779.

18. Simone M, Daunia V, Karen A, Donato N. Genetic variation and gastric cancer risk: a field synopsis and meta-analysis. Gut. 2015; 64:1209-1219.

19. Zhao Y, Chen F, Yang SJ, Tian Z, Wen YY, Xie Y, Pan XF, Marie L, Huang H, Lan H, Wen Y, Zhao ZM, Richie S, et al. Association of single nucleotide polymorphisms of CMA and ALDH2 with the risk of gastric cancer. Modern Preventive Medicine. 2014; 21:3975-3978.

20. Li SP, Ding JH, Cao HX, Wu JZ, Gao CM, Su P, Liu YT, Chang J, Yao GH. Alcohol dehydrogenase and aldehyde dehydrogenase genetic polymorphisms, alcohol consumption and the risk for gastric cancer. Chin J Prev Med. 2009; 43:644-626.

21. Yuan Q, Xue YD, Zheng YP, Wang QY. Investigation of ALDH2 gene polymorphisms and susceptibility to gastric cancer in the Chinese Han population in Jinhua, Zhejiang. Chin J Ctrl Endem Dis. 2016; 31:153-155.

22. Hidaka A, Sasazuki S, Matsuo K, Ito H, Sawada N, Shimazu T, Yamaji T, Iwasaki M, Inoue M, Tsugane S. Genetic polymorphisms of ADH1B, ADH1C and ALDH2, alcohol consumption, and the risk of gastric cancer: the Japan Public Health Center-based prospective study. Carcinogenesis. 2015; 36:223-231.

23. Yang S, Lee J, Choi IJ, Kim YW, Ryu KW, Sung J, Kim J. Effects of alcohol consumption, ALDH2 rs671 polymorphism, and Helicobacter pylori infection on the gastric cancer risk in a Korean population. Oncotarget. 2017; 24:6630-6641. https://doi.org/10.18632/oncotarget.14250.

24. Cao HX, Li SP, Wu JZ, Gao CM, Su P, Liu YT, Zhou JN, Ding JH. Alcohol dehydrogenase-2 and aldehyde dehydrogenase-2 genotypes, alcohol drinking and the risk for stomach cancer in Chinese males. Asian Pac J Cancer Prev. 2010; 11:1073-1077.

25. Chen ZH, Xian JF, Luo LP. Analysis of ADH1B Arg47His, ALDH2 Glu487Lys, and CYP4502E1 polymorphisms in gastric cancer risk and interaction with environmental factors. Genetics and Molecular Research. 2016; 15. https:// doi.org/10.4238/gmr15048904. 
26. Zhang LQ, Song X, Zhao XK, Huang J, Zhang P, Wang LW, Meng H, Ku JW, Kong GQ, Jiang T, Li XM, Lv XL, Ma T, et al. Association of genotypes of rs671 within ALDH2 with risk for gastric cardia adenocarcinoma in the Chinese Han population in high- and low-incidence areas. Cancer Biol Med. 2017; 14:60-65.

27. Chang SC, Chang PY, Butler B, Goldstein BY, Mu L, Cai L, You NC, Baecker A, Yu SZ, Heber D, Lu QY, Li L, Greenland S, et al. Single Nucleotide Polymorphisms of One-Carbon Metabolism and Cancers of the Esophagus, Stomach, and Liver in a Chinese Population. PLoS One. 2014; 9:e109235.

28. Chen CH, Ferreira JCB, Gross ER, Mochly-Rosen D. Targeting aldehyde dehydrogenase 2: New therapeutic opportunities. Physiol Rev. 2014; 94:1-34.

29. Duell EJ, Sala N, Travier N, Muñoz X, Boutron-Ruault MC, Clavel-Chapelon F, Barricarte A, Arriola L, Navarro C, Sánchez-Cantalejo E, Quirós JR, Krogh V, Vineis P, et al. Genetic variation in alcohol dehydrogenase (ADH1A, ADH1B, ADH1C, ADH7) and aldehyde dehydrogenase (ALDH2), alcohol consumption and gastric cancer risk in the European Prospective Investigation into Cancer and Nutrition (EPIC) cohort. Carcinogenesis. 2012; 33:361-367.

30. McKay JD, Truong T, Gaborieau V, Chabrier A, Chuang SC, Byrnes G, Zaridze D, Shangina O, Szeszenia-Dabrowska N, Lissowska J, Rudnai P, Fabianova E, Bucur A, et al. A genome-wide association study of upper aerodigestive tract cancers conducted within the INHANCE consortium. PLoS Genet. 2011; 7:e1001333.

31. Wu C, Kraft P, Zhai K, Chang J, Wang Z, Li Y, Hu Z, He Z, Jia W, Abnet CC, Liang L, Hu N, Miao X, et al. Genomewide association analyses of esophageal squamous cell carcinoma in Chinese identify multiple susceptibility loci and gene-environment interactions. Nat Genet. 2012; 44:1090-1097.
32. Boccia S, Hashibe M, Galli P, De FE, Asakage T, Hashimoto T, Hiraki A, Katoh T, Nomura T, Yokoyama A, van Duijn CM, Ricciardi G, Boffetta P. Aldehyde dehydrogenase 2 and head and neck cancer: a meta-analysis implementing a Mendelian randomization approach. Cancer Epidemiol Biomarkers Prev. 2009; 18:248-254.

33. Lewis SJ, Smith GD. Alcohol, ALDH2, and esophageal cancer: a meta-analysis which illustrates the potentials and limitations of a Mendelian randomization approach. Cancer Epidemiol Biomarkers Prev. 2005; 14:1967-1971.

34. Higgins JP, Thompson SG, Deeks JJ, Altman DG. Measuring inconsistency in meta-analyses. BMJ. 2003; 327:557-560.

35. DerSimonian R, Laird N. Meta-analysis in clinical trials. Control Clin Trials. 1986; 7:177-188.

36. Mantel N, Haenszel W. Statistical aspects of the analysis of data from retrospective studies of disease. J Natl Cancer Inst. 1995; 22:719-748.

37. Begg CB, Mazumdar M. Operating characteristics of a rank correlation test for publication bias. Biometrics. 1994; 50:1088-1101.

38. Egger M, Davey Smith G, Schneider M, Minder C. Bias in meta analysis detected by a simple, graphical test. BMJ. 1997; 315:629-634.

39. Hedges LV, Pigott TD. The power of statistical tests for moderators in meta-analysis. Psychol Methods. 2004; 9:426-445. 